\title{
Uso de autocuestionarios en reumatología. ¿Por qué su uso creciente y la necesidad de adaptarlos a cada población?
}

\section{Christian A. Waimann}

Médico especialista en Reumatología, Hospital Dr. Héctor Cura, Olavarría, Buenos Aires. Docente Universidad Nacional del Centro de la Pcia. de Buenos Aires.

Cualquier reumatólogo habituado a leer publicaciones y revisar presentaciones científicas en congresos, le puede haber llamado la atención la creciente cantidad de trabajos referentes al desarrollo y validación de autocuestionarios. Pero ¿por qué utilizar autocuestionarios y no medidas que incluyan sólo evaluación directa por parte del médico? Sin bien existen numerosas razones, se podrían resumir en dos principales. La primera es una necesidad básica de cuantificar dimensiones que sólo pueden ser autoevaluadas por el paciente. Un ejemplo clásico de esto es el dolor. Imagínense a ustedes como médicos explicándole al paciente: "En base a mi examen físico, me parece que su dolor es de 8 en 10”. Obviamente que el paciente lo miraría desorientado, y que esta medida carecería de toda validez, quedando atada a la subjetividad de cada médico. La otra gran razón para el uso de autocuestionarios es sistematizar evaluaciones y adecuarlas al tiempo y realidad de la consulta médica. Por ejemplo, se debe evaluar el impacto de una medicación sobre la capacidad funcional de un paciente. La forma más objetiva sería pedirle al paciente que realice una serie de actividades, graduando el médico el grado de limitación para cada una de ellas. Esto se enfrenta con dos realidades: el tiempo que insumiría lo haría inaplicable a la consulta diaria y por otro lado la evaluación de cada médico sería diferente y por lo tanto no comparable. Es por esta y otras razones que nacen los autocuestionarios, con el fin de estandarizar, sintetizar y permitir la evaluación de una variedad de dimensiones de la salud dentro de la consulta clínica y/o protocolos de investigación. Estos instrumentos nos permitieron, entre otras cosas, evaluar la respuesta a tratamientos, pronóstico, actividad y daño acumulado en los pacientes con diferentes enfermedades reumáticas.

Según las definiciones actuales, todas las dimensiones de la salud reportadas por los pacientes se engloban dentro del término "resultados percibidos por los pacientes" (Patient Reported Outcomes - PRO) definido por la Food and Drug Administration (FDA) como "cualquier medición de algún aspecto del estado de salud del paciente que sea realizada directamente por el paciente". Un concepto relacionado son los PROMs (Patient Reported Outcomes Measures) que representan las herramientas a partir del cual se recolecta la información, en este caso un autocuestionario. Y por último, ha surgido otro tipo de instrumento de evaluación por parte del paciente, que cuantifica las experiencias del mismo en el cuidado de su enfermedad y su interacción con el sistema de salud. A esta clase de autocuestionarios se los denomina PREMs (Patient Report Experiences Measures o "medidas de evaluación de experiencias reportadas por el paciente”). Ejemplos clásicos de esta última son los autocuestionarios de satisfacción con la atención médica ${ }^{1,2}$.

En nuestro país, se han traducido y validado un gran número de autocuestionarios incluyendo: HAQ (Health Assessment Questionnaire) ${ }^{3}$, HAQ-II ${ }^{4}$, PsAQoL (Psoriatic Arthritis Quality of Life) $)^{5}$, Rheumatoid Arthritis Impact of Disease (RAID) ${ }^{6}$, Indice Duruöz (ID) 7 , entre otros. Pero 
¿qué significa "Validar" un autocuestionario? En términos simples, para considerarse "válido" un cuestionario debe reunir las siguientes características:

- Validez y reproducibilidad. Implica que el autocuestionario cuantifique fehacientemente la dimensión para la cual fue diseñado, y que dicha medición sea reproducible bajo circunstancias similares.

- Capacidad de discriminación y sensibilidad al cambio. Es la habilidad del cuestionario para diferenciar entre diferentes estados de las dimensiones cuantificadas y registrar sus cambios en el tiempo.

- Aplicabilidad (“Feasibility”). Esto incluye consideraciones prácticas en su implementación, como son facilidad y tiempo para llenado, costo, equipamiento necesario, e interpretación de preguntas y resultados del cuestionario.

Cada una de estas propiedades se deben conservar en cada población donde se pretenda utilizar el autocuestionario. Siendo el concepto de población amplio, incluyendo características sociodemográficas (por ej., edad, sexo, etc.), patología (por ej., artritis reumatoidea, osteoartritis, etc.), y rasgos culturales (por ej., lenguaje formal e informal, etc.). Es de esta definición de población, que surge la necesidad de someter cada autocuestionario a un "proceso de validación" (corroborar que se conserven las propiedades del cuestionario), toda vez que la población difiera en alguna de sus características de la muestra original utilizada para el desarrollo del instrumento.

Uno de los autocuestionarios más difundidos actualmente en reumatología es el RAPID3. Este cuestionario fue desarrollado y validado inicialmente para evaluar la capacidad funcional y actividad de la enfermedad de pacientes con Artritis Reumatoidea. Su sencillez y velocidad de cálculo, fomentó su rápida difusión e implementación en la práctica diaria, validándose una versión adaptada a la población argentina9. Este autocuestionario evalúa tres dominios incluyendo capacidad funcional, dolor y valoración global del paciente sobre su enfermedad. Una de las características particulares de este instrumento es que a pesar de desarrollarse como una herramienta "específica” para Artritis Reumatoidea, las características abiertas de sus preguntas permiten la adaptación del mismo a otras patologías. Un ejemplo de esto, es la evaluación de la versión argentina del RAPID3 en pacientes con espondiloartropatía axial, evidenciando una adecuada reproducibilidad, capacidad discriminativa y aplicabilidad en dicha patología ${ }^{10}$.

Un caso particular ocurre en la Osteoartritis. A pesar de ser una de las patologías más prevalentes en nuestra especialidad, el uso de autocuestionarios está relativamente poco difundido en la práctica diaria. El instrumento por excelencia para la evaluación de estos pacientes es el Índice Western Ontario and McMaster Universities Osteoarthritis (WOMAC) ${ }^{11}$. Este consta de diferentes versiones, evaluando las dimensiones de dolor, rigidez y funcionalidad. Sin embargo, su longitud y falta de acceso libre (debe abonarse una patente para su uso), lo han relegado casi exclusivamente a la investigación clínica. Otras opciones disponibles y de acceso gratuito son el Knee and Hip injury and Osteoarthritis Outcome Score (KOOS y HOOS) ${ }^{12,13}$, pero su longitud (evalúan 5 dimensiones) y difícil cálculo reducen su aplicabilidad en la práctica diaria. Se han desarrollado versiones abreviadas, KOOSPS y HOOS-PS (KOOS y HOOS Physical function Short forms $)^{14}$, pero sólo evalúan capacidad funcional.

En este número, Kohan y cols. evaluaron en un grupo de pacientes con osteoartritis la correlación del RAPID3 con el WOMAC (osteoartritis de cadera y rodilla) y el Australian/ Canadian Osteoarthritis Index (AUSCAN; osteoartritis de manos) ${ }^{11}$. El RAPID3 resultó ser más sencillo y rápido para su llenado, presentando una muy buena correlación con funcionalidad de cadera y rodilla medida por WOMAC y regular correlación con dolor. El desempeño del RAPID3 en osteoartritis de manos fue menos satisfactorio, con una buena correlación con funcionalidad medida por AUSCAN y sin correlación con dolor. Los autores concluyen que el RAPID3 "podría ser una herramienta útil para evaluar pacientes con OA de manos, rodillas y/o caderas". Su utilidad estaría especialmente destinada a la evaluación de la capacidad funcional, necesitando valoraciones psicométricas adicionales a fin de determinar el comportamiento del cuestionario en pacientes con esta patología. 
En conclusión, los autocuestionarios continúan siendo una herramienta fundamental en nuestra práctica reumatológica diaria, siendo necesario que previo a la utilización de los mismos se apliquen los filtros de calidad correspondientes, a fin de aumentar la confiabilidad y validez de los mismos.

\section{Bibliografía}

1. Weldring T, Smith SM. Patient-Reported Outcomes (PROs) and Patient-Reported Outcome Measures (PROMs). Health Serv Insights. 2013;6:61-68.

2. Valderas JM, Alonso J. Patient reported outcome measures: a model-based classification system for research and clinical practice. Qual Life Res. 2008;17(9):1125-1135.

3. Citera G, Arriola MS, Maldonado-Cocco JA, et al. Validation and crosscultural adaptation of an argentine spanish version of the health assessment questionnaire disability index. J Clin Rheumatol. 2004;10(3):110-115.

4. Waimann CA, Citera G, Dal Para F, et al. Validación de una versión argentina del Health Assessment Questionnaire-II (HAQ-II). Rev Arg Reumatol. 2011;22(2):20-30.

5. Cazenave T, Maldonado Cocco JA, Citera G. Validación y adaptación cultural de una versión argentina del cuestionario para calidad de vida PsAQoL en pacientes con artritis psoriática. Rev Arg Reumatol. 2011;22(2):42-50.

6. Cayetti LA, Maldonado Ficco H, Schneeberger EE,et al. Validación del cuestionario Rheumatoid Arthritis Impact of Disease (RAID) en pacientes argentinos con artritis reumatoidea temprana y establecida. Rev Arg Reumatol. 2012;23(2):42-50.

7. Duarte V, Martire V, Crespo-Amaya G, et al. Validación del índice Duruöz en una población argentina con esclerodermia. Rev Arg Reumatol. 2015;26(2):23-26.

8. Wells G, Beaton DE, Tugwell P, et al. Updating the OMERACT filter: discrimination and feasibility. J Rheumatol. 2014;41(5):1005-1010.

9. Maldonado Ficco H, Pérez Alamino RS, Schneeberger EE, et al. Validación del cuestionario RAPID3 en una cohorte de pacientes con artritis reumatoidea temprana y establecida, y su correlación con otros índices de actividad. Rev Arg Reumatol. 2011;22(2):31-39.

10. Orozco MC, Schneeberger EE, Zamora N, et al. Validación del cuestionario RAPID3 en la cohorte argentina ESPAXIA de pacientes con espondiloartritis axial. Rev Arg Reumatol. 2016;27(4):11-16.

11. Bellamy N. The WOMAC Knee and Hip Osteoarthritis Indices: development, validation, globalization and influence on the development of the AUSCAN Hand Osteoarthritis Indices. Clin Exp Rheumatol. 2005;23(5 Suppl 39):S148-153.

12. Roos EM, Toksvig-Larsen S. Knee injury and Osteoarthritis Outcome Score (KOOS) - validation and comparison to the WOMAC in total knee replacement. Health Qual Life Outcomes. 2003;1:17.

13. Nilsdotter AK, Lohmander LS, Klässbo M, Roos EM. Hip disability and osteoarthritis outcome score (HOOS)--validity and responsiveness in total hip replacement. BMC Musculoskelet Disord. 2003;4:10.

14. Mehta SP, Sankar A, Venkataramanan V, et al. Crosscultural validation of the ICOAP and physical function short forms of the HOOS and KOOS in a multi-country study of patients with hip and knee osteoarthritis. Osteoarthritis Cartilage. 2016;24(12):2077-2081. 